\title{
O ÍNDICE DE ATKINSON E A SENSIBILIDADE DAS MEDIDAS DE DESIGUALDADE \\ A TRANSFERÊNCIAS REGRESSIVAS
}

\author{
Rodolfo Hoffmann*
}

\section{Resumo}

O artigo mostra como cstimar a desigualdade dentro dos cstratos quando o índicc de Atkinson ć calculado com basc cm uma tabcla de distribuição de freqücncias. Em seguida discutc-sc a sensibilidade das medidas de desigualdade a transferĉncias regressivas com dada razão cntrc as rendas, sugerindo-sc um critério mais restritivo que o de Shorrocks c Fostcr (1987).

\begin{abstract}
The paper shows how to estimate the incquality within classes when computing Atkinson's index from a frequency distribution tablc. The scnsitivity of incquality measures to regressive transfers with a given ratio between the two incomes is discussed and it is argued that this sensitivity should decrease with the income level, which is a morc restrictive critcria than the one proposed by Shorrocks and Fostcr (1987).
\end{abstract}

\section{Introdução.}

Os principais objetivos desse trabalho são: (1) mostrar como pode ser estimada a desigualdade dentro dos estratos quando o índice de Atkinson é calculado com base em uma tabela de distribuição de freqüências, sendo fornecida a renda média de cada estrato, e (2) discutir a sensibilidade das medidas de desigualdade a transferências regressivas.

A próxima seção trata' da definição e da interpretação do índice de Atkinson, e também da decomposição dessa medida de desigualdade quando a população é dividida em grupos. A seção 3 mostra como estimar a desigualdade dentro dos estratos quando o indice de Atkinson é calculado com base em uma tabela de distribuição de freqüências, ilustrando o procedimento com os dados da PNAD de

*ESALQ/USP. Uma versão anterior desse trabalho foi aprescntada no XV Encontro Brasilciro de Econometria, cm Bclo Horizontc, cm dez./1993.

R. de Econometria Rio cle Janeiro v. $14, n^{\circ} 2$, p.159-176 novembro $1994 /$ março 1995 
1989 sobre rendimento das pessoas ocupadas na agricultura brasileira. A última seção é dedicada à discussão da sensibilidade das medidas de desigualdade a transferências regressivas, sugerindo-se um critério mais restritivo que o de Shorrocks e Foster (1987), de maneira que essa sensibilidade seja uma função decrescente do nível de renda para transferências com dada razão entre as rendas.

\section{O Índice de Atkinson.}

Seja $x_{i}$

soas. Admite-se que a utilidade marginal da renda seja inversamente proporcional a $x_{i}^{\epsilon}$, isto é,

$$
\frac{d U\left(x_{i}\right)}{d x_{i}}=\frac{\Psi}{x_{\imath}^{\epsilon}}
$$

Impondo $\epsilon>0$ garante-se que a utilidade marginal diminua quando $x_{i}$

De (1) segue-se que, para $\epsilon \neq 1$,

$$
U\left(x_{i}\right)=\frac{\Psi}{1-\epsilon} x_{i}^{1-\epsilon}+C
$$

e, para $\epsilon=1$,

$$
U\left(x_{i}\right)
$$

Atkinson (1970) admite que o nível de bem estar social ( $W$ ) é uma função aditivamente separável e simétrica das rendas individuais, isto é,

$$
W=\sum_{i}^{n} U\left(x_{i}\right)
$$

De acordo com Atkinson (1970), o nível de renda equivalente numa distribuição igualitária $\left(x_{*}\right)$ é o valor da renda que cada pessoa deveria receber para, com todos recebendo renda igual, o nível de bem estar social ser igual ao correspondente à distribuição observada. Então 


$$
n U\left(x_{*}\right)=\sum_{i=1}^{n} U\left(x_{i}\right)
$$

A medida de desigualdade proposta por Atkinson é

$$
A(\epsilon)=1-\frac{x_{*}}{\mu}
$$

onde $\mu$ é a renda média da população:

$$
\mu=\frac{1}{n} \sum_{i=1}^{n} x_{i}
$$

Dada uma distribuição de renda com média $\mu$, a relação

$$
\frac{x_{*}}{\mu}=1-A(\epsilon)
$$

indica a proporção da renda total que seria suficiente para produzir o mesmo bem-estar social se a mesma renda total fosse igualitariamente distribuída.

De (2), (4) e (5) obtém-se, para $\epsilon \neq 1$,

$$
A(\epsilon)=1-\frac{1}{\mu}\left(\frac{1}{n} \sum x_{i}^{1-\epsilon}\right)^{\frac{1}{1-\epsilon}}
$$

De (3), (4) e (5) obtém-se, para $\epsilon=1$,

$$
A(\epsilon=1)=1-\frac{1}{\mu} \exp \left(\frac{1}{n} \sum \ln x_{i}\right)
$$

Pode-se verificar que:

a) O índice de Atkinson não é afetado por variações proporcionais na renda de todas as pessoas da população.

b) Para $\epsilon=0$ temos $A=0$, sempre.

c) No caso de uma distribuição perfeitamente igualitária $\left(x_{i}=\mu\right.$ para todo $i$ ), temos $A=0$, qualquer que seja o valor de $\epsilon$. 
d) Se $0<\epsilon<1$ e uma única pessoa se apropria de toda a renda $\left(x_{j}=n \mu\right.$ e $x_{i}=0$ para $\left.i \neq j\right)$, temos

$$
A=1-n^{-\frac{c}{1-c}}
$$

e) Quando $\epsilon \geq 1$, basta haver um indivíduo com renda nula para que tenhamos $A=1$. Isso corresponde a uma definição especial, onde identificamos o índice com o valor limite quando uma das rendas tende a zero, pois, a rigor, as funções (6) $\operatorname{com} \epsilon>1$ e (7) não são definidas para $x_{i}=0$.

f) Quando $\epsilon=10$ índice de Atkinson é a proporção em que a média geométrica das rendas é inferior à renda média.

g) Quando $\epsilon=2$ o índice de Atkinson é a proporção em que a média harmônica das rendas é inferior à renda média.

No índice de Atkinson, o parâmetro $\epsilon$ indica o grau de aversão à desigualdade: $\epsilon=0$ significa que há indiferença em relação à desigualdade, ao passo que valores elevados de $\epsilon$ significam forte aversão à desigualdade.

Para deixar mais claro o significado de $\epsilon$, Atkinson sugere um experimento mental envolvendo duas pessoas, uma com renda $x_{i}=M$ e outra com renda $x_{j}=2 M$. Subtrai-se $\$ 1$ da renda do mais rico e acrescenta-se $\$ \varphi$ à renda do mais pobre, com $0 \leq \varphi \leq 1$; é como se a fração $1-\varphi$ fosse gasta no processo de transferência. Para que as variações na utilidade das duas pessoas sejam iguais em valor absoluto devemos, de acordo com (1), ter

$$
\frac{\Psi}{(2 M)^{\epsilon}}=\frac{\Psi \varphi}{M^{\epsilon}}
$$

Segue-se que

$$
\varphi=\frac{1}{2^{\epsilon}}
$$

Se $\epsilon=1$ temos $\varphi=0,5$ e $1-\varphi=0,5$; a transferência aumenta o bem estar social desde que menos da metade da renda seja gasta na sua realização. Se $\epsilon=2$ temos $\varphi=0,25$ e $1-\varphi=0,75$, isto é, admite-se que os gastos com a transferência alcancem $3 / 4$ do valor subtraído do mais rico. Se $\epsilon=0$ temos $\varphi=1$ e qualquer gasto com a transferência fará com que o bem estar social diminua. 
Se a rencla da pessoa mais rica for 10 vezes maior do que a renda da mais pobre $\left(x_{i}=\Lambda\right.$ e $\left.x_{j}=10 M\right)$, a condição para igualdacle clas variações na utilidacle decorrentes da transferência fica

$$
\varphi=\frac{1}{10^{\epsilon}}
$$

Nesse caso, para $\epsilon=1$ temos $\varphi=0,1$, isto é, a transferência aumenta o bem estar social se mais de $10 \%$ da renda subtraícla do mais rico chegar ao mais pobre. Para $\epsilon=2$ temos $\varphi=0,01$, inclicando que a transferência cleveria ser realizada mesmo que quase $99 \%$ da rencla subtraícla do mais rico fosse gasta no processo.

Com base nessa análise parece-me razoável limitar os valores cle $\epsilon$ ao intervalo $(0,2)$. Valores de $\epsilon$ maiores do que 2 correspondem a níveis cle aversão à clesigualdacle extremamente elevaclos.

Em seguida será analisado o cálculo do índice de Atkinson para uma população dividida em grupos, obtendo-se expressões inclispensáveis para, na próxima seção, mostrar como se obtém o valor clo índice a partir cle uma tabela de distribuição de freqüências, incluinclo estimativas da clesigualdacle clentro dos estratos.

Considere-se uma população dividida em $k$ grupos e seja $x_{h i}$ a rencla da $i$-ésima pessoa do $h$-ésimo grupo $(h=1, \ldots, k ; i=$ $\left.1, \ldots, n_{k}\right)$. Seja $N=\Sigma n_{h}$ o número total de pessoas.

Se $\mu_{h}$ é a renda média da $h$-ésimo grupo, a média geral é

$$
\mu=\frac{1}{N} \sum_{h} n_{h} \mu_{h}
$$

Para $0 \leq \epsilon \neq 1$ o índice de Atkinson da população é

$$
A=1-\frac{1}{\mu}\left(\frac{1}{N} \sum_{h} \sum_{i} x_{h i}^{1-\epsilon}\right)^{\frac{1}{1-\epsilon}}
$$

e o índice de Atkinson dentro do h-ésimo grupo é

$$
A_{h}=1-\frac{1}{\mu_{h}}\left(\frac{1}{n_{h}} \sum_{i} x_{h i}^{1-\epsilon}\right)^{\frac{1}{1-\epsilon}}
$$


Verifica-se que o índice de Atkinson para a população pode ser expresso por (Nygard e Sandstrom, 1981, p. 330)

$$
A=1-\frac{1}{\mu}\left\{\frac{1}{N} \sum_{h} n_{h}\left[\left(1-A_{h}\right) \mu_{h}\right]^{1-\epsilon}\right\}^{\frac{1}{1-\epsilon}}
$$

Se $A_{h}=0$ para todo $h$, obtém-se o índice de Atkinson entre grupos:

$$
A_{c}=1-\frac{1}{\mu}\left(\frac{1}{N} \sum_{h} n_{h} \mu_{h}^{1-\epsilon}\right)^{\frac{1}{1-\epsilon}}
$$

Para $\epsilon=1$ o índice de Atkinson da população é

$$
A=1-\frac{1}{\mu} \exp \left(\frac{1}{N} \sum_{h} \sum_{i} \ln x_{h i}\right)
$$

e o ínclice clentro do $h$-ésimo grupo é

$$
A_{h}=1-\frac{1}{\mu_{h}} \exp \left(\frac{1}{n_{h}} \sum_{i} \ln x_{h i}\right)
$$

Verifica-se que o índice de Atkinson para a população pode ser expresso por

$$
A=1-\frac{1}{\mu} \exp \left\{\frac{1}{N} \sum_{h} n_{h} \ln \left[\left(1-A_{h}\right) \mu_{h}\right]\right\}
$$

e o ínclice entre grupos é

$$
A_{c}=1-\frac{1}{\mu} \exp \left(\frac{1}{N} \sum_{h} n_{h} \ln \mu_{h}\right)
$$

Cabe ressaltar que a diferença $A-A_{c}$ não é uma média ponclerada clos índices referentes à desigualdacle dentro dos grupos. 
3. Estiniação da desigualdade dentro de estratos no cálculo do indice de Atkinson.

Os dados disponíveis para o cálculo de medidas de desigualdade consistem, freqüentemente, no número de pessoas (ou famílias) e nos totais de renda para um certo número de estratos. Nestes casos, ao calcular uma medida da desigualdade da distribuição é aconselhável incorporar estimativas da desigualdade dentro de cada estrato.

Um dos métodos utilizados consiste em pressupor que a distribuição de renda dentro dos estratos com limite superior finito tem função de densidade linear e que no último estrato, sem limite superior finito, a distribuição é a de Pareto com dois parâmetros. A aplicação desse método na interpolação de percentis e no cálculo do índice de Gini e das medidas de desigualdade de Theil está descrita em Hoffmann (1979, 1984 e 1991). Aqui vamos estendê-lo ao cálculo do índice de Atkinson ${ }^{1}$.

Vamos admitir que dentro do estrato $(a, b)$ a distribuição de renda tenha média $\mu$ e função de densidade.

$$
f(x)=\alpha+\beta x
$$

Define-se

$$
\lambda=\frac{\mu-a}{b-a}
$$

Para $f(x) \geq 0$ no intervalo $[a, b)$ devemos ter $1 / 3 \leq \lambda \leq 2 / 3$. O procedimento a ser seguido caso essa condição não seja satisfeita é descrito em Hoffmann (1984).

Dados us valores de $a, b$ e $\mu$ podemos calcular $\lambda, \theta=b-a$,

$$
\beta=\frac{6(2 \lambda-1)}{\theta^{2}}
$$

e

$$
\alpha=\frac{1}{\theta}-\frac{\beta}{2}(a+b)
$$

${ }^{1}$ Kakwani (1980) apresenta vários métodos de estimar a desigualdade dentro de cstratos ao calcular medidas de desigualdade, mas considera, para o índice de Atkinson, apcnas os casos cm que $\epsilon=1$ c $\epsilon=2$. 
Para uma distribuição contínua as expressōes correspondentes a (6) e (7) são, respectivamente,

$$
A=1-\frac{1}{\mu}\left[\int_{a}^{b} x^{1-\epsilon} f(x) d x\right]^{\frac{1}{1-\epsilon}}
$$

e

$$
A=1-\frac{1}{\mu} \exp \left[\int_{a}^{b}(\ln x) f(x) d x\right]
$$

Uma vez que o cálculo referente à desigualdade dentro de cada estrato é feito tendo em vista determinar, em seguida, o índice de desigualdade para toda a população, de acordo com (10) ou (14), interessa obter, para cada estrato, o valor de

$$
B=[(1-A) \mu]^{1-\epsilon}=\int_{a}^{b} x^{1-\epsilon} f(x) d x \text { para } 0<\epsilon \neq 1
$$

ou

$$
B=\ln [(1-A) \mu]=\int_{a}^{b}(\ln x) f(x) d x \text { para } \epsilon=1
$$

Para o caso particular da função de densidade linear definida em (16), obtemos:

a) Para $0<\epsilon \neq 1, \epsilon \neq 2$ e $\epsilon \neq 1$

$$
B=\frac{\alpha}{2-\epsilon}\left(b^{2-\epsilon}-a^{2-\epsilon}\right)+\frac{\beta}{3-\epsilon}\left(b^{3-\epsilon}-a^{3-\epsilon}\right)
$$

b) Para $\epsilon=2$

$$
B=\alpha \ln \frac{b}{a}+\beta \theta
$$

c) Para $\epsilon=1$

$$
B=b(\ln b)\left(\frac{1}{\theta}-\frac{\beta a}{2}\right)-a(\ln a)\left(\frac{1}{\theta}-\frac{\beta b}{2}\right)-1+\frac{\beta \theta}{4}(a+b)
$$

Deixamos de apresentar a expressão para o caso particular de $\epsilon=3$ pois não vamos considerar valores de $\epsilon$ maiores do que 2 . 
Note-se que, para $\epsilon<2$, o valor da expressão (24) pode ser calculado mesmo quando $a=0$ (o que pode acontecer no primeiro estrato). No caso da Expressảo (25) $(\epsilon=2)$, com $\alpha>0$, temos

$$
\lim _{a \rightarrow 0^{+}} B=\infty
$$

e, lembrando (10), verifica-se que, nesse caso $a=0$ implica $A=1$. No caso da expressão $(26)(\epsilon=1)$ verifica-se que

$$
\lim _{a \rightarrow 0^{+}} B=\frac{b}{\theta} \ln b-1+\frac{\beta \theta b}{4}
$$

Quando o último estrato não tem limite superior finito, admitimos que dentro desse estrato a distribuição é a de Pareto com dois parâmetros, cuja funçâo de distribuição é

$$
F(x)=1-\left(\frac{a}{x}\right)^{\alpha}
$$

A média desta distribuição é

$$
\mu=\frac{\alpha a}{\alpha-1}
$$

Verifica-se que, para $\epsilon+\alpha>1$ e $\epsilon \neq 1$

$$
B=\frac{\alpha}{\epsilon+\alpha-1} a^{1-\epsilon}
$$

e para $\epsilon=1$

$$
B=\ln a+\frac{1}{\alpha}
$$

Com base nos limites e na renda média de cada estrato podemos, assim, obter o respectivo valor de $B$. Em seguida, utilizando também o número de pessoas em cada estrato, podemos calcular o índice de Atkinson da população através de (10) ou (14).

Para ilustrar a aplicação do método vamos utilizar os dados da PNAD de 1989 referentes à distribuição das pessoas ocupadas na agricultura brasileira, classificadas de acordo com seu reindimento 


\section{Tabela 1.}

Pessoas ocupadas na agricultura brasileira e rendimento médio para 13 estratos de rendimento mensal de todos os trabalhos, de acordo com a PNAD de 1989.

\begin{tabular}{|c|c|c|}
\hline $\begin{array}{l}\text { Limites do cstrato } \\
\text { cm salários mínimos }\end{array}$ & $\begin{array}{l}\text { Númcro } \\
\text { pessoas }\end{array}$ & $\begin{array}{r}\text { Rendimento médio } \\
\text { (NCZ\$) }\end{array}$ \\
\hline $\begin{array}{l}\text { Atć } 0,25 \\
\text { Mais dc } 0,25 \text { a } 0,5 \\
\text { Mais dc } 0,5 \text { a } 0,75 \\
\text { Mais dc } 0,75 \text { a } 1 \\
\text { Mais dc } 1 \text { a } 1,5 \\
\text { Mais dc } 1,5 \text { a } 2 \\
\text { Mais de } 2 \text { a } 3 \\
\text { Mais dc } 3 \text { a } 5 \\
\text { Mais dc } 5 \text { a } 7 \\
\text { Mais dc } 7 \text { a } 10 \\
\text { Mais dc } 10 \text { a } 15 \\
\text { Mais dc } 15 \text { a } 20 \\
\text { Mais clc } 20\end{array}$ & $\begin{array}{r}679702 \\
1452163 \\
1210200 \\
1671526 \\
1811251 \\
810353 \\
983323 \\
697310 \\
231657 \\
185931 \\
137250 \\
13600 \\
131136\end{array}$ & $\begin{array}{r}12,51 \\
99,70 \\
155,81 \\
220,24 \\
291,82 \\
118,56 \\
574,31 \\
916,94 \\
1167,18 \\
1995,10 \\
2895,76 \\
1185,39 \\
10476,24\end{array}$ \\
\hline Total & 10112038 & 545,82 \\
\hline
\end{tabular}

${ }^{1}$ Salário mínimo no mês de referĉncia da PNAD (sctcmbro de 1989): NCZ\$ 219,18.

todos os trabalhos, excluindo as pessoas ocupadas sem rendimento do trabalho. Cabe lembrar que o levantamento da PNAD não abrange a área rural da Região Norte. A amostra inclui 18.304 observações que, de acordo com os pesos fornecidos pelo IBGE, correspondem a 10.112.038 pessoas ocupadas na agricultura cujo rendimento de todos os trabalhos é positivo.

A tabela 1 mostra a distribuição de freqüências dessas pessoas, considerando 13 estratos de rendimento de todos os trabalhos, e o rendimento médio em cada estrato.

Também foi considerada uma distribuição de freqüências com apenas 6 estratos cujos limites inferiores são 0, 0,5, 1, 2, 5 e 10 salários mínimos. Esses são os estratos utilizados na publicação dos resultados da PNAD (ver IBGE, 1991, p. 16).

Utilizando o método descrito nesta seção, o índice de Atkinson foi calculado, para vários valores de $\epsilon$, utilizando as informações da tabela 1 (13 estratos) ou considerando informações para apenas 6 
Tabela 2.

Medidas da desigualdade da distribuição do rendimento de todos os trabalhos entre pessoas ocupadas na agricultura brasileira, excluindo as pessoas com rendimento igual a zero, de acordo com a PNAD de 1989.



estratos. Para avaliar a exatidão do método, o índice de Atkinson também foi calculado utilizando os dados individuais. Os resultados estão na tabela 2, juntamente com valores do índice de Gini e das medidas de desigualdade de Theil, calculadas nas mesmas condições.

Se considerarmos que o valor dessas medidas de desigualdade geralmente é arredondado na terceira casa decimal, pode-se afirmar que os valores obtidos a partir da tabela de distribuição de freqüências estão muito próximos dos valores exatos, mesmo quando temos informações para apenas 6 estratos. A aproximação é especialmente boa para valores de $\epsilon$ "intermediários", surgindo diferenças um pouco maiores para valores de $\epsilon$ relativamente baixos $(\epsilon=0,25)$ ou relativamente elevados $(\epsilon=1,5$ ou $\epsilon=2)$. Essas diferenças maiores parecem estar associadas a uma maior sensibilidade do índice ao que ocorre nas caudas da distribuição. Note-se que a diferença entre o valor estimado com base na tabela de distribuição de freqüências e o valor exato também é relativamente elevada no caso da redundância, que é uma medida de desigualdade bastante sensível ao que ocorre na cauda superior da distribuição. 
Cabe lembrar que o índice de Atkinson para $\epsilon=1$ é uma função do $L$ de Theil $(L)$ :

$$
A(\epsilon=1)=1-\exp (-L)
$$

4. Sensibilidade a transferências regressivas.

Una transferência regressiva de rencla consiste em subtrair um montante da rencla de uma pessoa e acrescentá-lo à de uma outra pessoa que, anteriormente, já tinha renda maior do que a primeira. Portanto, uma transferência regressiva sempre aumenta a diferença entre as rendas das duas pessoas envolvidas. O princípio de PigouDalton estabelece que o valor de uma medida cle desigualdacle da distribuição da renda deve aumentar quando é feita uma transferência regressiva.

Vamos admitir que as pessoas estão ordenaclas da mais pobre até a mais rica, isto é,

$$
x_{1} \leq x_{2} \leq \ldots \leq x_{n}
$$

Se $x_{h}<x_{j}$, uma transferência regressiva consiste em subtrair $\omega>0$ de $x_{h}$ e aclicioná-lo a $x_{j}$.

A sensibiliclade do índice de Atkinson a uma transferência regressiva é clacla por

$$
\frac{d A}{d \omega}=\frac{1}{n} \mu^{\epsilon-1}(1-A)^{\epsilon}\left[\frac{1}{\left(x_{h}-\omega\right)^{\epsilon}}-\frac{1}{\left(x_{j}+\omega\right)^{\epsilon}}\right]
$$

Como essa expressão aincla clepende de $\omega$, para facilitar as comparações vamos considerar seu limite quando $\omega$ tencle a zero; isto é, vamos consiclerar

$$
\lim _{\omega \rightarrow 0} \frac{d \cdot A}{(l \omega}=\lim _{\omega \rightarrow 0} \frac{\Delta A}{\omega}=\frac{1}{n} \mu^{\epsilon-1}(1-A)^{\epsilon}\left(\frac{1}{x_{h}^{\epsilon}}-\frac{1}{x_{j}^{\epsilon}}\right)
$$

Para analisar como o efeito da transferência regressiva varia com o nível de renda das pessoas envolvidas é usual fixar a diferença $\left(x_{j}-\right.$ $x_{h}$ ) entre essas renclas. Aqui vamos fixar a razão entre elas, isto é, vamos considerar

$$
x_{j}=\beta x_{h}, \operatorname{com} \beta>1
$$


Nesse caso obtemos

$$
\lim _{\omega \rightarrow 0} \frac{\Delta A}{\omega}=\frac{\mu^{\epsilon-1}(1-A)^{\epsilon}\left(\beta^{\epsilon}-1\right)}{n x_{h}^{\epsilon} \beta^{\epsilon}}
$$

A opção por considerar transferências regressivas com dada diferença ou com dada razão entre elas é, até certo ponto, arbitrária, não afetando as principais conclusões sobre a sensibilidade das diferentes medidas de desigualdade a transferências regressivas. Pode-se argumentar, entretanto, que considerar rendas com dada razão é mais coerente com a noção usual de desigualdade. Note-se que todas as medidas mais comuns de desigualdade não sofrem alteração se todas as rendas são multiplicadas por uma constante, uma operação que também não afeta a razão entre duas rendas quaisquer, mas altera a diferença entre elas.

A expressão (32) mostra que a sensibilidade do índice de Atkinson a transferências regressivas é inversamente proporcional a $x^{\epsilon}$. A diminuição da sensibilidade quando a renda aumenta (para $\epsilon>0$ ) é tanto mais rápida quanto maior o valor de $\epsilon$. O próprio Atkinson (1970, p. 59) já observou que "quando $\epsilon$ cresce, associamos mais peso a transferências na cauda inferior da distribuição e menos peso a transferências na parte superior".

A seguir vamos construir um gráfico comparando a sensibilidade relativa do índice de Gini $(G)$, da redundância $(R)$, do coeficiente de variação $(C)$ e do índice de Atkinson (para vários valores de $\epsilon$ ) a transferências regressivas com $x_{j} / x_{h}=\beta>1$. As expressões para a sensibilidade do índice de Gini, da redundância e do coeficiente de variação podem ser encontradas em Hoffmann (1992).

Uma vez que a sensibilidade do índice de Gini depende da densidade de probabilidade da distribuição, vamos considerar uma distribuição de renda log-normal com média e variância dos logarítimos iguais a $-0,7$ e 1,5 , respectivamente, semelhante à distribuição da renda entre pessoas, no Brasil, em 1980, classificadas de acordo com seu rendimento familiar per capita (em salários mínimos de agosto de 1980 por pessoa) (ver Hoffmann, 1992).

As curvas qué mostram a sensibilidade relativa de $G, R, C$ e $A(\epsilon)$ a transferências regressivas estão na figura 1. Conisidera-se um 
valor de $\beta=x_{j} / x_{h}$ pouco superior a 1 , para que a sensibilidade do índice de Gini possa ser associada à densidade de probabilidade do logaritmo da renda. Como a finalidade é comparar a sensibilidade relativa das medidas de desigualdade, as curvas foram construídas de maneira que seu valor médio no intervalo de $x_{h}=0,04$ a $x_{h}=10$ fosse aproximadamente igual a 100 para todas as medidas de desigualdade (embora, para maior clareza, o rendimento máximo considerado na figura 1 seja apenas 8 salários mínimos per capita). Cabe assinalar que o valor de $\beta$ não afeta a posição das curvas, desde que seja bastante próximo de 1. É indiferente, por exemplo, considerar $\beta=1,01$ ou 1,001. Embora o valor do efeito da transferência regressiva sobre as medidas de desigualdade diminua quando $\beta$ diminui, o comportamento das sensibilidades relativas é estável para valores de $\beta$ próximos de 1 .

Devido à relação (31), a curva de sensibilidade relativa do $L$ de Theil é idêntica à curva relativa ao índice de Atkinson para $\epsilon=1$.

A figura 1 mostra que a existência da "aversão à desigualdade" de Atkinson $(\epsilon>0)$ corresponde a curvas de sensibilidade relativa decrescentes. Com $\epsilon>0$ a sensibilidade do índice de Atkinson a transferências regressivas diminui com o nível de renda, quando se fixa a razão entre as rendas afetadas pela transferência. A redundância representa uma espécie de "fronteira": uma medida de desigualdade cuja sensibilidade relativa independe do nível de renda.

$\mathrm{Na}$ análise de Shorrocks e Foster (1987) sobre a sensibilidade das medidas de desigualdade a transferências regressivas a "fronteira" é dada pelo comportamento do coeficiente de variação. Isso porque aqueles autores consideraram, inicialmente, transferências com uma dada diferença $\left(\alpha=x_{j}-x_{h}\right)$ entre as rendas envolvidas. Para esse tipo de transferência regressiva a sensibilidade do coeficiente de variação independe do nível de renda.

Para deixar mais clara a diferença entre o critério de Shorrocks e Foster (1987) e aquele sugerido pela figura 1, é interessante considerar a família de índices de Shorrocks²:

$$
S=\frac{1}{k(k-1)}\left(\frac{1}{n \mu^{k}} \sum x_{i}^{k}-1\right)
$$

${ }^{2}$ Ver Ramos e Barros (1989, p.10). 
Rodolfo Hoffmann

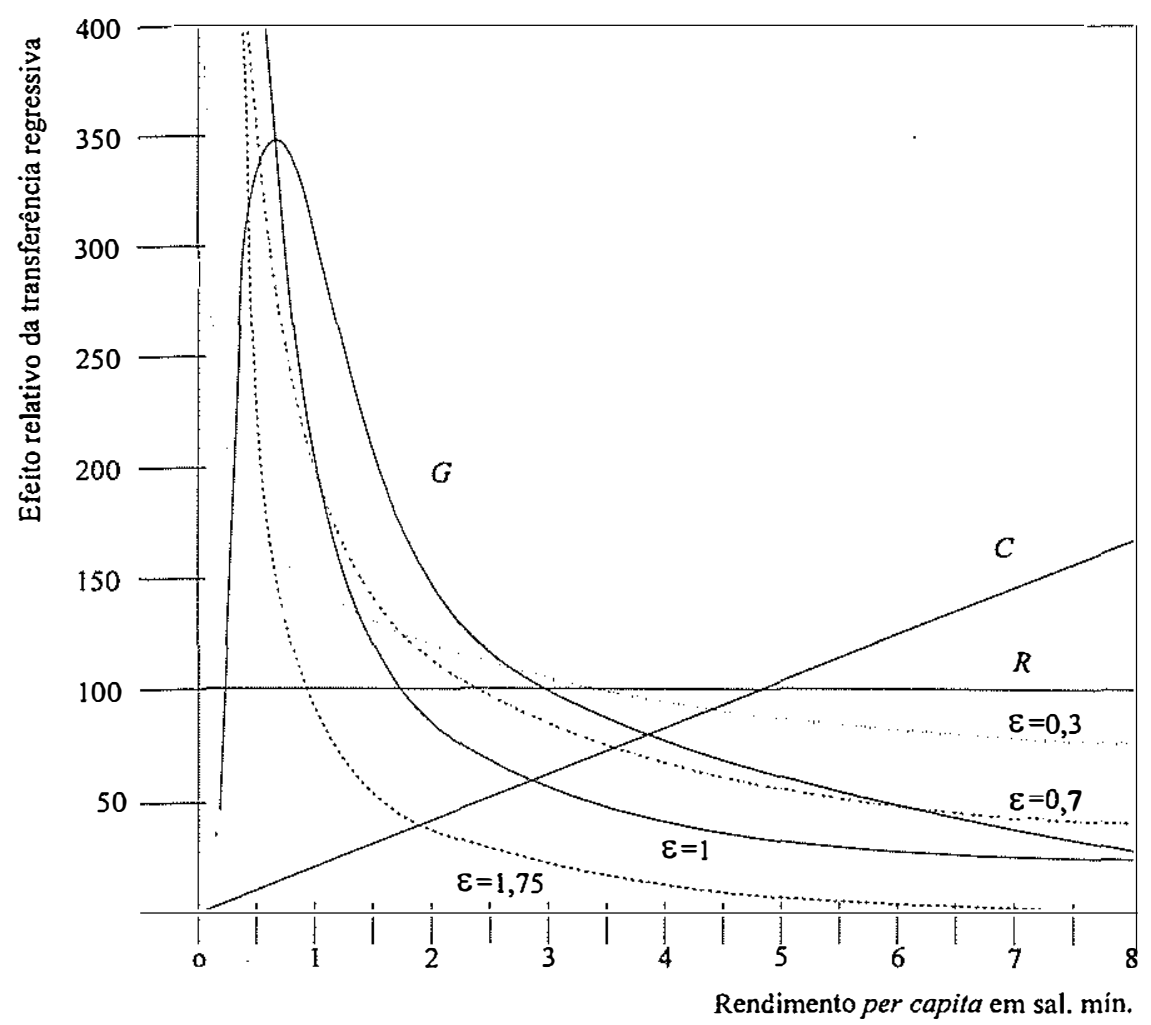

Figura 1.

Sensibilidade relativa de várias medidas de desigualdade a transferências regressivas com dada razão entre as rendas: indice de Gini $(G)$, redundância $(R)$, coeficiente de variação $(C)$ e indice de Atkinson para $\epsilon=0,3, \epsilon=0,7, \epsilon=1$ e $\epsilon=1,75$.

O coeficiente de variação $(C)$, a redundância $(R)$ e o $L$ de Theil $(L)$ correspondem a casos particulares do índice de Shorrocks, pois

$$
C=\sqrt{2 S}
$$

R. de Econometria 14(2) novembro 1994/março 1995 
quando o parâmetro $k$ é igual a 2 ,

$$
R=\lim _{k \rightarrow 1} S
$$

$\mathrm{e}$

$$
L=\lim _{k \rightarrow 0} S
$$

De acordo com Shorrocks e Foster (1087) o índice $S$ apresenta sensibilidade decrescente se $k<2$.

Se considerarmos transferências regressivas com dada razão entre as rendas, a sensibilidade de $S$ só é decrescente se $k<1$.

A sensibilidade de $S$ a transferências regressivas com dada razão entre as rendas $\left(\beta=x_{j} / x_{h}\right)$ é dada por

$$
\lim _{\omega \rightarrow 0} \frac{\Delta S}{\omega}=\frac{1-\beta^{k-1}}{n(1-k) \mu^{k} x_{h}^{1-k}}
$$

Fazendo $k=1-\epsilon$, verifica-se que, para $k<1$ (correspondendo a $\epsilon>0$ ), a curva de sensibilidade relativa do índice de Shorrocks é idêntica à do índice de Atkinson.

Em resumo, a "aversão à desigualdade" de Atkinson corresponde, na figura 1, a curvas de sensibilidade decrescente. O coeficiente de variação e o índice de Shorrocks $\operatorname{com} k>1$ são medidas de desigualdade cuja sensibilidade a transferências regressivas com dada razão entre as rendas aumenta com o nível de renda.

Isso não significa que o coeficiente de variação deva ser totalmente descartado como medida de desigualdade. É possível que seja a medida mais adequada, se estivermos especialmente interessados em ressaltar mudanças na cauda superior da distribuição.

Nas situaçōes usuais, entretanto, parece recomendável utilizar medidas de desigualdade cuja sensibilidade a transferências regressivas com dada razão entre as rendas não cresça com nível de renda. Levando em consideração o que foi discutido na seção 2, pode-se limitar o valor do parâmetro $\epsilon$ do indice de Atkinson ao intervalo $[0,2]$ e, portanto, limitar o valor do parâmetro $k$ do índice de Shorrocks ao intervalo $[-1,1]$. A redundância (que corresponde a $k=1$ ) está em um dos limites do intervalo adotado; para essa medida de desigualdade a sensibilidade a transferências regressivas com dada razão 
entre as rendas independe do nível de renda. Um indice de Shorrocks com $k=1,5$, que satisfaz o critério de Shorrocks e Foster (1987), já seria considerado "excessivamente" sensivel a transferências na cauda superior da distribuição pelo critério ora proposto.

A sensibilidade do índice de Gini a transferências regressivas com dada razão entre as rendas é proprocional à densidade de freqüência relativa dos logaritmos das rendas que, no caso de uma distribuição log-normal, é máxima para a renda mediana (ver Hoffmann, 1992). Haverá, então, usualmente, um intervalo, para rendas relativamente baixas, em que a sensibilidade do índice de Gini cresce com o nível de renda, como mostra a figura 1. Isso não é razão para deixar de considerar o índice de Gini uma boa medida de desigualdade. A figura 1 mostra que, excluindo as rendas muito próximas de zero, a sensibilidade do índice de Gini a transferências regressivas é substancialmente maior para rendas relativamente baixas (em torno da mediana) do que para rendas elevadas. Pode-se argumentar, inclusive, que a pouca sensibilidade do índice de Gini ao que ocorre com rendas muito próximas de zero é uma qualidade desejável na medida em que tais rendas correspondem a situações em que o daclo não corresponde à renda real da pessoa. Esse é o caso, por exemplo, das pessoas ocupadas que são membros não remunerados das famílias de pequenos agricultores, cuja renda declarada é igual a zero. Note-se que o índice de Gini pode ser utilizado para comparar distribuições onde há pessoas com renda igual a zero, ao passo que um índice especialmente sensível ao que ocorre na cauda inferior da distribuição, como um índice de Atkinson $\operatorname{com} \epsilon \geq 1$, é inútil para uma comparação desse tipo, pois basta haver uma renda igual a zero para que 0 indice já assuma seu valor máximo $(A=1)$.

\section{Referências}

Atkinson, A. B. 1970. "On the measurement of inequality." Journal of Economic Theory 2:244-263. O artigo é reproduzido, juntamente com um apêndice não-matemático, in Atkinson, A. B. (org.), (1973) Wealth, Income and Inequality. Penguin Books, pp. 46-68.

Hoffmann, R. 1979. "Estimação da desigualdade dentro de estratos 
no cálculo do índice de Gini e da redundância." Pesquisa e Planejamento Econômico 9:719-738.

1984. "Estimation of inequality and concentration measures from grouped observations." Revista de Econometria $4: 5-21$.

1991. "O índice de desigualdade de Theil-Atkinson." R.rin.str de Econometria 11:143-160.

1992. "Sensibilidade das medidas de desigualdade a transferências regressivas." Pesquisa e Planejamento Econômico 22: $289-304$.

IBGE. 1991. "Pesquisa nacional por amostra de domicílios." vol. 13, 1989. Tomo 1 - Brasil e Grandes Regiões.

Kakwani, N. C. 1980. Income Inequality and Poverty: methods of estimation and policy applications. Banco Mundial. Oxford: Oxford University Press.

Nygard, F. \& A. Sandstrom 1981. Measuring Income Inequality. Estocolmo: Almqvist \& Wiksell International.

Ramos, L. R. A. \& R. P. Barros 1989. "Medidas de desigualdade." Texto apresentado na terceira Escola de Séries Temporais e Econometria. Rio de Janeiro: IPEA-INPES.

Shorrocks, A. F. \& J. E. Foster 1987. "Transfer sensitive inequality measures." Review of Economic Studies 54:485-497. 